NOUVELLE

\section{Mutations des gènes impliqués dans l'épissage dans les hémopathies malignes humaines}

Frederik Damm ${ }^{1,2,3}$, Florence Nguyen-Khac ${ }^{4,5}$, Olivier Kosmider ${ }^{6,7,8}$, Michaela Fontenay ${ }^{6,7,8}$, Olivier A. Bernard 1,2,3,6
${ }^{1}$ Institut Gustave Roussy, 114, rue Édouard Vaillant, 94805

Villejuif Cedex, France ;

${ }^{2}$ Inserm U985, Villejuif, France ;

3 université Paris Sud, Orsay, France ;

${ }^{4}$ service d'hématologie biologique, centre hospitalier PitiéSalpêtrière, Assistance publique-hôpitaux de Paris (APHP), Paris, France ;

${ }^{5}$ Inserm U872, université Pierre et Marie Curie, Paris, France ; ${ }^{6} \mathrm{APHP}$, service d'hématologie biologique, hôpital Broca-

Cochin-Hôtel-Dieu, Paris, France ;

${ }^{7}$ département d'immunologie et hématologie, Institut Cochin, Paris, France ;

${ }^{8}$ Inserm U1016, CNRS UMR8104, université Paris Descartes,

Paris, France.

olivier.bernard@inserm.fr
Génétique des hémopathies malignes: les apports des techniques de séquençage massif

Le développement des techniques de séquençage massif bouleverse notre compréhension des processus de transformation à l'origine des hémopathies malignes. Ces approches permettent d'identifier de nombreuses mutations acquises dans des maladies malignes dont les bases moléculaires étaient jusqu'à aujourd'hui mal connues. L'analyse des conséquences prévisibles de ces mutations a permis d'identifier des processus biologiques dont la dérégulation participerait aux mécanismes de transformation.

Dans une série de travaux publiés récemment, les séquences codantes de l'ADN obtenu à partir des cellules mononucléées de la moelle osseuse de patients souffrant d'un syndrome myélodysplasiquel (SMD) ont été comparées à celles de l'ADN de cellules considérées comme exemptes de la maladie (lymphocytes T ou prélèvement jugal) [1-3]. Cette approche a permis d'identifier en moyenne 10 mutations acquises par échantillon et de confirmer la fréquence des mutations déjà connues dans cette maladie. Elle a révélé

${ }^{1}$ Les myélodysplasies représentent un groupe hétérogène d'hémopathies acquises, caractérisées par des degrés variables d'hématopoï̀se inefficace et par une probabilité importante de transformation leucémique. II existe plusieurs classifications basées sur des critères morphologiques (frottis de moelle osseuse) et cytogénétiques. Les signes cliniques et leur gravité sont en rapport avec la nature et l'intensité de la cytopénie : anémie, complications infectieuses, hémorragies. l'existence de mutations touchant des gènes dont les produits sont impliqués dans le contrôle du mécanisme d'épissage des ARN messagers (Tableau I). Les mutations de cette catégorie de gènes sont trouvées dans 45 à $85 \%$ des SMD, et sont le plus souvent mutuellement exclusives. La majorité des mutations observées sont des mutations faux-sens, situées dans des régions restreintes des protéines. Les mutations du gène SF3BI sont observées plus fréquemment dans les SMD avec sidéroblastes en couronne ${ }^{2}$. Ces mutations de SF3BI ont été décrites comme étant un critère de bon pronostic indépendant dans les SMD, mais ce point n'est pas consensuel $[2,4,5]$.

Des analyses similaires ont été réalisées dans la leucémie lymphoïde chronique (LLC). Dans cette maladie, le génome de cellules tumorales - des lymphocytes $B\left(D 19^{+} C D^{+}\right.$- a été comparé à celui de cellules contrôles non tumorales, soit granuleuses, soit fibroblastiques $[6,7]$. Dans une première publication, des mutations de onze gènes ont été identifiées de façon récurrente, dont certains déjà connus dans cette pathologie, tels que TP53 et ATM (voir Glossaire) (Tableau II). Les prédictions fonctionnelles montrent que ces gènes

${ }^{2}$ On appelle sidéroblastes des érythroblastes qui accumulent du fer non héminique dans les mitochondries. Quand au moins les deux tiers du pourtour du noyau sont entourés par ces mitochondries, on parle de sidéroblastes en couronne. Ils sont visibles après coloration de Perls. interviennent normalement dans cinq processus cellulaires: la réparation des dommages de l'ADN et le cycle cellulaire, la voie de signalisation Wnt, la voie de signalisation NOTCHI, les voies de signalisation de l'inflammation, et les mécanismes de contrôle de l'épissage [6]. Lorsque les échantillons présentent des mutations des régions hypervariables des gènes codant pour les chaînes lourdes des immunoglobulines (IGHV) remaniés, le nombre de mutations acquises modifiant la séquence codante est supérieur (12,8 par échantillon) à ce qu'il est dans les échantillons dépourvus de ces mutations somatiques (10,6 par échantillon) [7]. Des mutations des gènes POTI dont le produit est impliqué dans la biologie des télomères -, CHD2 - dont le produit est impliqué dans le contrôle de la structure chromatinienne -, et $\angle R P I B$ - un gène candidat suppresseur de tumeur -, allongent la liste des gènes fréquemment mutés dans les LLC.

Ces résultats pourraient avoir une application clinique. En effet, les mutations de certains gènes semblent associées aux critères cliniques ou biologiques caractéristiques de la LLC. Par exemple, les mutations de NOTCHI et de FBXW7 sont associées à la présence d'une trisomie 12 et à un statut non muté des IGHV [6]. Les mutations de MYD88 sont associées au caractère muté des IGHV et aux délétions de la région chromosomique $13 q 14$ [6]. 


\begin{tabular}{|c|c|c|c|c|c|c|}
\hline Gène & $\begin{array}{l}\text { SMD sans } \\
\text { sidéroblastes } \\
\text { en couronne }\end{array}$ & $\begin{array}{l}\text { SMD avec } \\
\text { sidéroblastes } \\
\text { en couronne }\end{array}$ & SMP & LMMC & LAM & LLC \\
\hline SF3BI & $6,5 \%(10 / 155)$ & $75,3 \%(55 / 73)$ & $0 \%(0 / 53)$ & $4,5 \%(4 / 88)$ & $3,2 \%(7 / 213)$ & $9,7 \%(27 / 279)$ \\
\hline SRSF2 & $11,6 \%(18 / 155)$ & $5,5 \%(4 / 73)$ & $1,9 \%(1 / 53)$ & $28,4 \%(25 / 88)$ & $2,3 \%(5 / 213)$ & \\
\hline ZRSR2 & $7,7 \%(12 / 155)$ & $1,4 \%(1 / 73)$ & $1,9 \%(1 / 53)$ & $8 \%(7 / 88)$ & $0,5 \%(1 / 213)$ & $1 \%(1 / 105)$ \\
\hline U2AF35 & $\begin{array}{c}11,6 \%(18 / 155) \\
8,7 \%(13 / 150)\end{array}$ & $0 \%(0 / 73)$ & $1,9 \%(1 / 53)$ & $8 \%(7 / 88)$ & $3,8 \%(8 / 213)$ & \\
\hline U2AF65 & $0,6 \%(1 / 155)$ & $0 \%(0 / 73)$ & $0 \%(0 / 53)$ & $1,1 \%(1 / 88)$ & $0 \%(0 / 213)$ & $1,9 \%(2 / 105)$ \\
\hline SF3Al & $1,3 \%(2 / 155)$ & $0 \%(0 / 73)$ & $0 \%(0 / 53)$ & $1,1 \%(1 / 88)$ & $0,9 \%(2 / 213)$ & \\
\hline SFI & $1,3 \%(2 / 155)$ & $0 \%(0 / 73)$ & $1,9 \%(1 / 53)$ & $0 \%(0 / 88)$ & $0 \%(0 / 213)$ & \\
\hline PRPF4OB & $1,9 \%(3 / 155)$ & $0 \%(0 / 73)$ & $1,9 \%(1 / 53)$ & $0 \%(0 / 88)$ & $0,9 \%(2 / 213)$ & \\
\hline NXFI & $4,7 \%(1 / 21)$ & & & $0 \%(0 / 4)$ & $0 \%(0 / 7)$ & $2,8 \%(3 / 105)$ \\
\hline XPO1 & $\begin{array}{c}12,5 \%(1 / 8) \\
0 \%(0 / 21)\end{array}$ & & & & & $\begin{array}{c}2,4 \%(4 / 165) \\
1,9 \%(2 / 105) \\
1 \%(1 / 91)\end{array}$ \\
\hline
\end{tabular}

Tableau I. Mutations des gènes codant pour des protéines impliquées dans la biologie de l'ARN dans les hémopathies myéloïdes et les LLC. Données provenant des références $[1,3,6,7]$. SMD : syndrome myélodysplasique; SMP : syndrome myéloprolifératif ; LMMC : leucémie myélomonocytaire chronique ; LAM : leucémie aiguë myéloïde; LLC : leucémie lymphoïde chronique. Pour la dénomination des gènes, voir Glossaire.

Les mutations de SF3BI sont associées aux délétions de la région chromosomique 1lq22 (et aux mutations du gène ATM qui est localisé dans cette région), à un mauvais pronostic et une résistance à la fludarabine.

\section{Nouveaux gènes impliqués}

dans les LLC et les SMD :

quels enseignements pour

la compréhension de la

physiopathologie de ces hémopathies?

\section{Les LLC partagent des mutations}

\section{avec les autres pathologies lymphoïdes}

Des mutations de NOTCHI sont connues depuis plusieurs années dans les leucémies aiguës lymphoblastiques T (LAL-T) ; elles se traduisent par une activation constitutive de la transcription des gènes cibles de cette voie [8]. Durant sa maturation, la protéine $\mathrm{NOTCH}$ est clivée en deux parties : une partie extracellulaire $(\varepsilon C N)$ et une partie intracellulaire (ICN) qui interagissent entre elles. L'interaction de NOTCHI avec ses ligands libère ICN qui migre dans le noyau où elle interagit avec le facteur de transcription RBPJ $\kappa$ et active les gènes cibles de la voie. Dans les LAL-T, les mutations de NOTCHI peuvent soit faciliter la dissociation de la partie intracellulaire ICN de NOTCHI, soit entrầner la perte du domaine PEST de déstabilisation de ICN. Ce sont des mutations de ce dernier type qui sont présentes dans les LLC, principalement une mutation «saut de phase » touchant le codon correspondant à l'acide aminé P2515 [9-11]. Des mutations de NOTCHI sont également trouvées dans les lymphomes. Comme pour les LAL-T, des mutations inactivatrices du gène $F B X W 7$ - qui exerce normalement un rétrocontrôle sur la voie NOTCHI - sont aussi retrouvées dans les LLC [6].

Des mutations ponctuelles de MYD88 ont déjà été décrites dans des lymphomes de type B. Il s'agit systématiquement de mutations faux-sens de type activateur. Les mutations de MYD88 entraînent l'activation des facteurs de transcription STAT3 et NFkB. La mutation L265P décrite dans les LLC est largement majoritaire dans les lymphomes de type $B$.

\section{Implication récurrente de gènes dont les produits participent à la biologie de I'ARN}

La surprise de cette série de résultats récents est l'identification de mutations dans des gènes dont les produits sont impliqués dans le contrôle de l'épissage de l'ARN. L'implication d'oncogènes dans les mécanismes de maturation de l'ARN est connue depuis longtemps. On peut citer 0TT/RBMI5 qui est impliqué dans l'export des ARN messagers vers le cytoplasme et remanié dans les translocations $t(1 ; 22)(p 13 ; q 13)$ des leucémies 
à mégacaryoblastes $[12,25]$. Un autre exemple est l'implication des protéines du nucléopore, NUP98 et NUP214, dans des protéines de fusion créées par des remaniements géniques [13].

Dans les SMD, 8 gènes codant pour des protéines impliquées dans l'épissage sont mutés avec une fréquence variable $[1,2]$. Hormis les mutations inactivatrices qui touchent - presque uniquement chez les hommes - le gène SRSF2 localisé sur le chromosome $X$, les autres mutations sont des mutations faux-sens qui s'accumulent à haute densité dans des régions spécifiques de ces gènes, affectant très souvent un acide aminé unique. Ce type de profil de mutation indique souvent un gain de fonction. Ces mutations pourraient affecter l'efficacité de l'assemblage des splicéosomes ou de l'épissage luimême. L'expression de la plupart des gènes pourrait être affectée du fait du maintien inopportun d'introns dans les transcrits matures, de l'omission d'exons, ou de la dérégulation des épissages alternatifs. Le rôle de l'épissage alternatif dans la différenciation cellulaire a été établi dans plusieurs exemples et la dérégulation de ces processus pourrait participer à la transformation cellulaire $[14,15](\rightarrow)$. Les mutations affectant l'épissage

$(\rightarrow)$ Voir $\mathrm{m} / \mathrm{s} \mathrm{n}^{\circ} 4$, avril 2012, pages 372 et 381

pourraient également entraîner une variation soit de l'efficacité de la transcription en amont, soit de la cinétique de l'export vers le cytoplasme en aval. Les vitesses de transcription, d'épissage et de transport vers le cytoplasme sont liées et influencent la structure chromatinienne [16]. Schématiquement, un ralentissement (ou une accélération) de ces processus pourrait aboutir à la fermeture (ou à l'ouverture) de la chromatine indépendamment de la régulation normale, et donc à des anomalies de la différenciation cellulaire.

Il est possible que des altérations touchant d'autres aspects de la biologie de l'ARN jouent également un rôle dans les processus de transformation. Dans les LLC, des mutations de CPSF2, $D D X 3 X$ et XPO1 (qui code pour l'exportine 1) ont été décrites. Le produit de CPSF2 est impliqué dans les processus de polyadénylation de l'ARN messager, les protéines de la famille des DEADbox sont impliquées dans la biologie des ARN et XPOl participe à l'export nucléocytoplasmique de protéines et d'ARN.

Des mutations affectant des gènes dont les produits participent au contrôle de la traduction ou de la stabilité des ARNm ont été décrites dans le myélome, un autre type de tumeur de la lignée lymphoïde B [17]. Des mutations de DIS3 (qui code pour une sous-unité catalytique des exosomes) ont été décrites : elles semblent être des mutations inactivatrices, associées à la perte de l'autre copie du gène. Ces mutations pourraient aboutir à une accumulation de certains ARNm et interférer avec le contrôle de la traduction. Des mutations de DIS3 ont par ailleurs été décrites dans des LAM (leucémies aiguës myéloblastiques) [18] et dans deux échantillons de LLC [6]. Des mutations de LRRK2, dont la protéine est impliquée, entre autres, dans le contrôle de la traduction par ses capacités à phosphoryler le facteur d'initiation de la traduction $4 \varepsilon B P$, ont été rapportées. De plus, le gène $F A M 46 C$ - qui est également muté dans le myélome - jouerait un rôle dans la traduction ou la stabilité de I'ARN.

Ces données sont bien sûr très récentes, mais il est curieux de constater que plusieurs étapes de la biologie de l'ARN, maturation, transport, traduction et dégradation, semblent être ciblées dans les processus de transformation tumorale. Elles s'ajoutent à la description du rôle de la perte du gène RPS14, codant pour une protéine ribosomique, dans la pathogenèse des SMD avec $5 q$ [19] et un rôle potentiel des ARN non codants dans le contrôle de l'épissage [16].

\section{Les mutations du gène $S F 3 B 1$} sont fréquentes dans les LLC et les SMD Le gène $S F 3 B 1$, qui code pour une protéine impliquée dans l'épissage, est muté dans 10 à $15 \%$ des SMD et une proportion équivalente de LLC. L'identification d'un gène muté à la fois dans les SMD et les LLC est inattendue, tant ces deux pathologies sont considérées comme éloignées sur le plan physiopathologique. Les SMD sont considérées comme des maladies de la cellule souche, tandis que les LLC sont considérées comme résultant de la transformation de cellules engagées dans la différenciation lymphoïde de type B. Une explication évidente est que les mutations de SF3BI ont les mêmes conséquences dans les deux types de cellules, et donc participent aussi bien à la transformation d'un lymphocyte $B$ qu'à celle d'un progéniteur myéloïde. L'observation de mutations de SF3BI dans d'autres tumeurs, incluant des syndromes myéloprolifératifs et des tumeurs solides, plaide en faveur de cette hypothèse $[2,20]$. SF3Bl pourrait participer à l'activité des polycomb repressive complex.

Des données récentes indiquent que les LLC pourraient dériver d'anomalies des cellules souches hématopoïétiques [21]. Un autre argument vient des études récentes du gène TET2 [26]. Les mutations de TET2 peuvent être observées dans des hémopathies myéloïdes malignes et dans des hémopathies lymphoïdes matures, principalement lymphoïdes T. Dans ces deux cas, il est possible de mettre en évidence la présence de ces mutations dans les progéniteurs myéloïdes, suggérant également que ces pathologies pouvant avoir pour origine une cellule souche [22].

Une partie de ces données pourrait rapidement trouver une application clinique du fait d'un lien établi entre ces mutations, la présentation clinique au diagnostic et le pronostic. De plus, SF3Bl est déjà la cible d'agents thérapeutiques, tels que la splicéostatine $A$ [23]. Curieusement, les conséquences 


\begin{tabular}{|c|c|c|c|c|}
\hline Gène & Fréquence & Fonction & Remarque & Références \\
\hline TP53 & $15 \%(15 / 91)$ & Contrôle des dommages à l'ADN et du cycle cellulaire & NGS & [6] \\
\hline SF3B1 & $15 \%(14 / 91)$ & Métabolisme de l'ARN & NGS & [6] \\
\hline SF3BI & $9,5 \%(10 / 105)$ & & NGS & [7] \\
\hline SF3B1 & $5 \%(17 / 301)$ & & Échantillons successifs & {$[24]$} \\
\hline SF3BI & $17 \%(10$ of 59$)$ & & $\begin{array}{l}\text { Échantillons résistants } \\
\text { à la fludarabine }\end{array}$ & [24] \\
\hline MYD88 & $10 \%(9 / 91)$ & Inflammation et voies de signalisation NFKB et JAK & NGS & {$[6]$} \\
\hline MYD88 & $2,8 \%(3 / 105)$ & & NGS & {$[7]$} \\
\hline ATM & $9 \%(9 / 91)$ & Contrôle des dommages à l'ADN et du cycle cellulaire & NGS & [6] \\
\hline ATM & $3,8 \%(4 / 105)$ & & NGS & {$[7]$} \\
\hline NOTCHI & $4 \%(4 / 91)$ & Voie Notch & NGS & {$[6]$} \\
\hline NOTCHI & $4,7 \%(5 / 105)$ & & NGS & {$[7]$} \\
\hline NOTCHI & $8,3 \%$ & & Échantillons au diagnostic & {$[10]$} \\
\hline NOTCHI & $(31,0 \%)$ & & Transformation (Richter) & {$[10]$} \\
\hline NOTCHI & $(20,8 \%)$ & & Échantillons chimiorésistants & {$[10]$} \\
\hline FBWX7 & $4 \%(4 / 91)$ & Voie Notch & NGS & {$[6]$} \\
\hline Zмумз & $4 \%(4 / 91)$ & Histone déacétylase - structure chromatinienne & NGS & {$[6]$} \\
\hline ZMYмз & $1,9 \%(2 / 105)$ & & $\begin{array}{l}\text { NGS un exemple de mutation } \\
\text { dans ZMYMI }\end{array}$ & {$[7]$} \\
\hline DDX3X & $3 \%(3 / 91)$ & Métabolisme de l'ARN & NGS & {$[6]$} \\
\hline DDX3X & $1,9 \%(2 / 105)$ & Métabolisme de l'ARN & NGS & {$[7]$} \\
\hline MAPK1 & $3 \%(3 / 91)$ & Inflammation et voies de signalisation NFKB et JAK & NGS & {$[6] \mid$} \\
\hline CHD2 & $4,7 \%(5 / 105)$ & Hélicase - structure chromatinienne & NGS & [7] \\
\hline CHD2 & $1 \%(1 / 91)$ & & NGS & {$[6]$} \\
\hline POT1 & $4,7 \%(5 / 105)$ & Biologie des télomères & NGS & {$[7]$} \\
\hline POT1 & $1 \%(1 / 91)$ & & NGS & [6] \\
\hline$A S X L 1$ & $2,8 \%(3 / 105)$ & Polycomb & NGS & {$[7]$} \\
\hline$A S X L 1$ & $1 \%(1 / 91)$ & & NGS & {$[6]$} \\
\hline NXF1 & $2,8 \%(3 / 105)$ & Métabolisme de l'ARN & NGS & {$[7]$} \\
\hline SFRS1 & $1,9 \%(2 / 105)$ & Métabolisme de l'ARN & NGS & [7] \\
\hline U2AF65 & $1,9 \%(2 / 105)$ & Métabolisme de l'ARN & NGS & {$[7]$} \\
\hline U2AF65 & $1 \%(1 / 91)$ & & NGS & {$[6]$} \\
\hline XPO1 & $1,9 \%(2 / 105)$ & Métabolisme de l'ARN & NGS & [7] \\
\hline XPO1 & $1 \%(1 / 91)$ & & NGS & [6] \\
\hline
\end{tabular}

Tableau II. Principaux gènes mutés dans les LLC. NGS : next generation sequencing ; les gènes indiqués en gras ont une fonction définie. Dénomination des gènes, voir Glossaire. 


\section{GLOSSAIRE}

ASXL1 : additional sex combs like 1

ATM : ataxia telangiectasia mutated

CHD2 : chromodomain helicase DNA binding protein 2

CPSF2 : cleavage and polyadenylation specificity factor subunit 2

DDX3X : DEAD (Asp-Glu-Ala-Asp) box polypeptide 3, X-linked

FAM46C : family with sequence similarity 46

FBXW7 : F-box/WD repeat-containing protein 7

LAL-T : leucémies aiguës lymphoblastiques T

LAM : leucémies aiguës myéloblastiques

LLC : leucémie lymphoïde chronique

LRRK2 : leucine-rich repeat kinase 2

LRPIB : low density lipoprotein receptor-related protein $1 B$

MAPK1 : mitogen-activated protein kinase 1

MYD88 : myeloid differentiation primary response gene

NUP98 et NUP214 : nuclear pore complex protein

NXF1 : nuclear RNA export factor 1

0TT/RBM15 : one twenty-two / RNA binding motif protein 15

POTl : protection of telomere protein 1

PRPF40B : pre-mRNA processing factor 40 homolog B

RPS14 : ribosomal protein S14

SF3B1, SF1 : splicing factor

SMD : syndrome myélodysplasique

SMP : syndrome myéloprolifératif

SRSF2 : serine/arginine (SR)-rich splicing factor

TहT2 : tet oncogene family member 2

U2AF65, U2AF35 : U2 small nuclear riboprotein auxiliary factor 65 or 35

XPO1 : code pour l'exportine 1

ZRSR2 : zinc finger (CCCH type), RNA-binding motif and serine/arginine rich 2

ZMYM3 : zinc finger MYM-type 3

cellulaires de ces mutations semblent être néfastes pour la croissance cellulaire. La compréhension des mécanismes d'action de ces mutations est essentielle pour le développement de thérapies ciblées, mais nécessitera des analyses fonctionnelles plus poussées et le développement de modèles sophistiqués. $\diamond$

Mutations in genes involved in splicing in human malignancies

\section{LIENS D'INTÉRÊT}

Les auteurs déclarent n'avoir aucun lien d'intérêt concernant les données publiées dans cet article.

\section{REMERCIEMENTS}

Frederik Damm bénéficie du soutien financier de la Deutsche Krebshilfe. Les travaux des auteurs sont financés par l'Inserm, la direction de la recherche clinique AP-HP (PHRC MDS-04), l'institut national du cancer (INCa), l'association Laurette Fugain et une labellisation de la ligue nationale contre le cancer (LNCC).

\section{RÉFÉRENCES}

1. Yoshida K, Sanada M, Shiraishi Y, et al. Frequent pathway mutations of splicing machinery in myelodysplasia. Nature $2011 ; 478: 64-9$.

2. Papaemmanuil $\varepsilon$, Cazzola M, Boultwood J, et al. Somatic SF3B1 mutation in myelodysplasia with ring sideroblasts. N Engl J Med 2011 ; 365 : 1384-95.

3. Graubert TA, Shen D, Ding L, et al. Recurrent mutations in the U2AFl splicing factor in myelodysplastic syndromes. Nat Genet 2011 ; 44 53-7.

4. Patnaik MM, Lasho TL, Hodnefield JM, et al. SF3B1 mutations are prevalent in myelodysplastic syndromes with ring sideroblasts but do not hold independent prognostic value. Blood 2012 ; 119 : 569-72.

5. Damm F, Thol F, Kosmider 0, et al. SF3Bl mutations in myelodysplastic syndromes: clinical associations and prognostic implications. Leukemia 2011 ; doi: 10.1038/leu.2011.321.

6. Wang L, Lawrence MS, Wan Y, et al. SF3Bl and other novel cancer genes in chronic lymphocytic leukemia. N Engl J Med 2011 ; 365 : 2497-506.
7. Quesada V, Conde L, Villamor N, et al. Exome sequencing identifies recurrent mutations of the splicing factor SF3Bl gene in chronic lymphocytic leukemia. Nat Genet 2011 ; 44 : 47-52.

8. Ferrando AA. The role of NOTCHI signaling in T-ALL. Hematology Am Soc Hematol Educ Program 2009: 353-61.

9. Di lanni $M$, Baldoni $S$, Rosati $\varepsilon$, et al. A new genetic lesion in B-CLL: a NOTCHI PEST domain mutation. $\mathrm{Br}$ J Haematol 2009 ; 146 : 689-91.

10. Fabbri G, Rasi S, Rossi D, et al. Analysis of the chronic lymphocytic leukemia coding genome: role of NOTCHI mutational activation. J Exp Med 2011 ; 208 : 1389-401.

11. Puente XS, Pinyol M, Quesada V, et al. Whole-genome sequencing identifies recurrent mutations in chronic lymphocytic leukaemia. Nature 2011 ; 475 : 101-5.

12. Hiriart $\varepsilon$, Gruffat $H$, Buisson $M$, et al. Interaction of the Epstein-Barr virus mRNA export factor $\varepsilon B 2$ with human Spen proteins SHARP, OTIl, and a novel member of the family, $0 \Pi 3$, links Spen proteins with splicing regulation and mRNA export. J Biol Chem 2005 ; 280 : 36935-45.

13. Xu S, Powers MA. Nuclear pore proteins and cancer. Semin Cell Dev Biol 2009; $20: 620$-30.

14. Christofk HR, Vander Heiden MG, Harris MH, et al. The M2 splice isoform of pyruvate kinase is important for cancer metabolism and tumour growth. Nature $2008 ; 452$ : 230-3.

15. Gabut M, Samavarchi-Tehrani P, Wang X, et al. An alternative splicing switch regulates embryonic stem cell pluripotency and reprogramming. Cell 2011 ; 147 : 132-46.

16. Luco RF, Misteli T. More than a splicing code: integrating the role of RNA, chromatin and noncoding RNA in alternative splicing regulation. Curr Opin Genet Dev 2011 ; 21: 366-72.

17. Chapman MA, Lawrence MS, Keats JJ, et al. Initial genome sequencing and analysis of multiple myeloma. Nature 2011 ; 471 : 467-72.

18. Ding L, Ley TJ, Larson DE, et al. Clonal evolution in relapsed acute myeloid leukaemia revealed by wholegenome sequencing. Nature 2012 ; 481 : 506-10.

19. Ebert BL. Molecular dissection of the $5 q$ deletion in myelodysplastic syndrome. Semin Oncol 2011 ; 38 : 621-6.

20. Lasho TL, Finke CM, Hanson CA, et al. SF3Bl mutations in primary myelofibrosis: clinical, histopathology and genetic correlates among 155 patients. Leukemia 2011.

21. Kikushige $Y$, Ishikawa F, Miyamoto T, et al. Selfrenewing hematopoietic stem cell is the primary target in pathogenesis of human chronic lymphocytic leukemia. Cancer Cell 2011; $20: 246-59$.

22. Quivoron C, Couronne L, Della Valle V, et al. TET2 inactivation results in pleiotropic hematopoietic abnormalities in mouse and is a recurrent event during human lymphomagenesis. Cancer Cell $2011 ; 20: 25-38$.

23. Yokoi A, Kotake Y, Takahashi K, et al. Biological validation that SF3b is a target of the antitumor macrolide pladienolide. FEBS J 2011 ; 278 : 4870-80.

24. Rossi D, Bruscaggin A, Spina V, et al. Mutations of the SF3Bl splicing factor in chronic lymphocytic leukemia: association with progression and fludarabinerefractoriness. Blood $2011 ; 118: 6904-8$.

25. Bernard OA, Gilliland DG, Mercher T. Activation de la voie Notch par OTT-MAL dans les leucémies aiguës mégacaryoblastiques. Med Sci (Paris) 2009 ; 25 : 676-8.

26. Mercher T, Quivoron C, Couronné L, et al. I In cauda venenum ou de l'importance de (la) TET(2). Med Sci (Paris) $2011 ; 27: 1064-6$. 